\title{
Changes in the Electrical Properties of Supraoptic Nucleus Oxytocin and Vasopressin Neurons during Lactation
}

\author{
Javier E. Stern and William E. Armstrong \\ Department of Anatomy and Neurobiology, College of Medicine, University of Tennessee, Memphis, Tennessee 38163
}

Magnocellular oxytocin (OT) and vasopressin (VP) neurons adopt different firing patterns in response to relevant physiological stimuli. OT neurons selectively display short (2-4 sec), high-frequency bursts of action potentials that are highly synchronized and correlated with OT release during lactation. The present experiments were done to determine whether the electrophysiological properties of OT neurons differ from those of VP neurons, and whether these properties are modulated during lactation to support short bursting activity. Intracellular recordings in vitro were obtained from immunochemically identified supraoptic neurons of diestrous or lactating female rats. Resting membrane potential, input resistance, membrane time constant, and the depolarizing afterpotential did not differ among groups. However, near spike threshold, OT, but not VP, neurons expressed a sustained outward rectification that was removed by small hyperpolarizing pulses and a rebound depo- larization that occurred at the offset of these hyperpolarizing pulses. The rebound depolarization was short $(<2$ sec), supported brief bursts of action potentials, and was significantly larger during lactation. Neurons expressing the outward rectification also exhibited strong spike frequency adaptation during prolonged (1-4 sec) depolarization. Spike width, the $\mathrm{Ca}^{2+}$ dependent afterhyperpolarization, and the degree of spike broadening of OT, but not VP, neurons were also larger during lactation, suggesting an increase in $\mathrm{Ca}^{2+}$ influx per spike. The results indicate that OT neurons possess properties favoring the expression of short spike trains, and that some of these properties are enhanced during lactation. In addition, spikes in OT neurons may promote more $\mathrm{Ca}^{2+}$ influx in this state.

Key words: electrophysiology; oxytocin; vasopressin; lactation; supraoptic nucleus; magnocellular cells
Oxytocin (OT) and vasopressin (VP) hormone release from nerve terminals in the neurohypophysis is correlated with the electrical activity of magnocellular neuroendocrine cells (MNCs) located in the supraoptic (SON) and paraventricular nuclei of the hypothalamus. During hemorrhage or osmotic stimulation, VP neurons increase their firing rate and eventually adopt an asynchronous phasic bursting pattern characterized by trains of impulses $(8-15$ $\mathrm{Hz}$ ) lasting tens of seconds and separated by silent intervals of similar duration (Brimble and Dyball, 1977; Poulain et al., 1977). On the other hand, OT neurons during lactation display a synchronized, brief $(2-4 \mathrm{sec})$, high-frequency $(40-80 \mathrm{~Hz})$ burst of activity preceding each milk ejection (Wakerley and Lincoln, 1973). The electrical activity of OT neurons also is increased during cardiovascular and osmotic stimuli, but in these cases OT cells fire continuously rather than in bursts (Brimble and Dyball, 1977; Poulain et al., 1977; Wakerley et al., 1978).

The efficiency of stimulus-secretion coupling is regulated both by the degree and the pattern of electrical activity of MNCs. Previous studies using the isolated neurohypophysis have shown that when the gland is subjected to higher frequencies of electrical stimulation, hormone release becomes potentiated (Dreifuss et al,

\footnotetext{
Received Feb. 14, 1996; revised May 16, 1996; accepted May 21, 1996.

This work was supported by National Institutes of Health (NIH) Grant NS23941 to W.E.A. and by the Neuroscience Center for Excellence in the Department of Anatomy and Neurobiology, University of Tennessee, Memphis, TN. We thank Mr. Emin Kuliyev for valuable technical assistance and Dr. Robert C. Foehring for commenting on this manuscript. We are deeply indebted to Dr. Alan G. Robinson for providing antiserum to VP-neurophysin (generated under NIH Grant AM16166) and to Drs. Harold Gainer and Mark Whitnall, who provided the antibody PS36.

Correspondence should be addressed to Dr. Javier E. Stern, Department of Anatomy and Neurobiology, College of Medicine, University of Tennessee, 855 Monroe Avenue, Memphis, TN 38163.

Copyright (C) 1996 Society for Neuroscience $0270-6474 / 96 / 164861-11 \$ 05.00 / 0$
}

1971). Furthermore, the amount of peptide secreted per impulse is modulated by the pattern of neural activity (Dutton and Dyball, 1979; Bicknell et al., 1982; Cazalis et al., 1985). During hemorrhage or dehydration, the asynchronous bursting pattern of VP neurons facilitates a sustained antidiuresis. Thus, the amount of peptide released per impulse during the initial stages of a burst is enhanced, and the secretory fatigue at the axon terminal is being minimized by the silent periods in the phasic pattern (Bicknell, 1988). This enhancement is also present for OT release, which also shows less fatigue when subjected to continuous stimulation of the neural lobe than does VP release (Bicknell, 1988).

The pattern of electrical activity generally results from the interaction of intrinsic ionic mechanisms and synaptic activity. In the case of VP neurons, there is direct evidence that the phasic bursting activity arises from an intrinsic regenerating mechanism involving the summation of depolarizing afterpotentials (DAPs) that support a plateau potential underlying the burst (Andrew and Dudek, 1983, 1984a). However, the origin of the burst in OT neurons during lactation is unknown. There is no detailed data so far on the intrinsic electrophysiological properties of OT neurons during lactation. The present experiments were done to determine whether the electrical properties of OT neurons change during lactation in a manner that might favor short bursting activity. In a previous study, we have shown that OT, but not VP, neurons displayed a sustained outward rectification (SOR) and a rebound depolarization (RD), which might favor the expression of short burst of action potentials (Stern and Armstrong, 1995a). The objectives of the present study were to characterize in detail the intrinsic membrane properties and firing responses of SON OT and VP neurons in female rats and to evaluate the effects of lactation on their expression. 

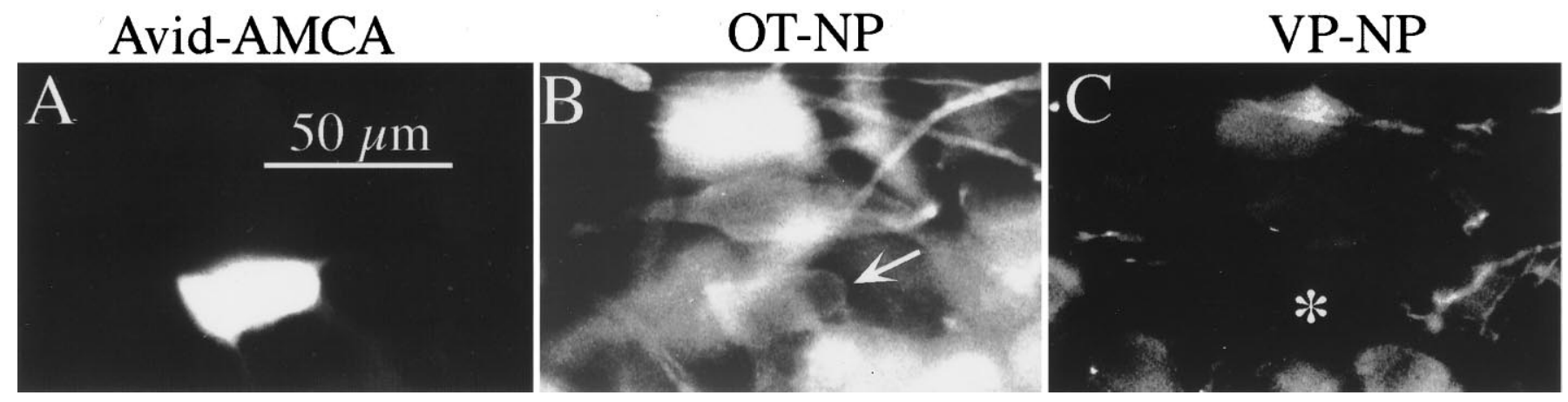

Figure 1. Example of an immunochemically identified OT neuron. The neuron was intracellularly labeled with neurobiotin and immunochemically labeled for VP- and OT-associated neurophysin by double immunofluorescence in a $50 \mu \mathrm{m}$ vibratome section. $A$, The neurobiotin-injected neuron is visualized by AMCA-conjugated avidin. B, OT-neurophysin immunoreactivity visualized by tetramethylrhodamine-conjugated secondary antibody. Arrow points to the recorded cell. $C$, VP-neurophysin immunoreactivity visualized by FITC-conjugated secondary antibody. The recorded neuron was not immunoreactive, as marked by the asterisk.

Some of the results have appeared in abstract form (Armstrong and Stern, 1995).

\section{MATERIALS AND METHODS}

Explant preparation. Female virgin diestrous rats (assessed by vaginal smear the morning of the experiment) and lactating albino rats (200-390 gm; Holtzman, Harlan Laboratories, Indianapolis, IN) that had suckled for $8-14 \mathrm{~d}$ by at least 10 pups were used as subjects. The rats were anesthetized with sodium pentobarbital $(50 \mathrm{mg} / \mathrm{kg}$, i.p.) and perfused through the heart with cold medium in which $\mathrm{NaCl}$ was replaced by an equiosmolar amount of sucrose (Aghajanian and Rasmussen, 1989). A ventral hypothalamic explant was removed and placed in an incubation chamber as described previously (Smith and Armstrong, 1990). The incubation medium consisted of (in $\mathrm{mM}$ ): $25 \mathrm{NaHCO}_{3}, 3 \mathrm{KCl}, 1.24$ $\mathrm{NaH}_{2} \mathrm{PO}_{4}, 124 \mathrm{NaCl}, 10$ glucose, $2 \mathrm{CaCl}_{2}, 1.3 \mathrm{MgCl}_{2}, 0.2$ ascorbic acid, 0.2 thiourea. The antioxidant compounds increased substantially the number of viable neurons recorded from these older adult animals (Rice and Nicholson, 1991). The medium was saturated with $95 \% \mathrm{O}_{2} / 5 \% \mathrm{C}_{2}$ with a $\mathrm{pH}$ of 7.3-7.4 and an osmolarity of $290-300 \mathrm{mOsm} / \mathrm{kg} \mathrm{H} \mathrm{H}_{2}$, and was warmed to $32-34^{\circ} \mathrm{C}$. All chemicals, unless otherwise stated, were purchased from Sigma (St. Louis, MO).

Electrophysiology. Intracellular recordings, signal digitization, and data analysis were made as described previously (Smith and Armstrong, 1990; Armstrong et al., 1994). Briefly, intracellular recording electrodes were pulled from $1.5 \mathrm{~mm}$ glass pipettes on a Sutter horizontal puller. The direct current resistance of the electrodes ranged between 70 and 150 $\mathrm{M} \Omega$. For intracellular labeling, microelectrodes were filled with $1-2 \mathrm{M}$ potassium acetate containing $2 \% \mathrm{~N}$-(2-aminoethyl)biotinamide (Neurobiotin; Vector Laboratories, Burlingame, CA) (Kita and Armstrong, 1991). Intracellular injections were made with $200 \mathrm{msec}, 0.5 \mathrm{nA}$ rectangular pulses at $1 \mathrm{~Hz}$ for at least $10 \mathrm{~min}$. Recordings were obtained with a Neurodata amplifier. Continuous direct current recordings were displayed on a chart recorder and digitized at $44 \mathrm{kHz}$ for storage on videotape (Neurocorder; from Neurodata). In addition, traces were acquired at different rates using the Labmaster TL-1 in conjunction with pClamp 6 software (Axon Instruments, Foster City, CA). All neurons considered had membrane potentials of $-50 \mathrm{mV}$ or more negative and action potentials of at least $55 \mathrm{mV}$. Current-voltage $(I-V)$ plots were generated by passing $180 \mathrm{msec}$ pulses through the electrode. Apparent membrane resistance was taken as the slope of a linear regression measured at the linear part of the curve. Membrane time constant was estimated using exponential fits of the voltage transient generated from a short $(5 \mathrm{msec})$ current pulse sufficient to hyperpolarize the membrane $10-15 \mathrm{mV}$ from rest. To measure action potential properties, neurons were current-clamped close to threshold, and a $5 \mathrm{msec}$, $0.1 \mathrm{nA}$ depolarizing pulse was applied. Spike height was measured from the beginning of the fastest rising phase to the peak of the action potential, whereas spike width was measured at $50 \%$ of the peak from threshold. Current-evoked firing behavior was evaluated by measuring successive interspike intervals during a $180 \mathrm{msec}$ pulse using different current injection amplitudes.

Immunohistochemistry. Fixation and sectioning of the explants were done as described previously (Armstrong et al., 1994). Briefly, after the recording session the explants were fixed in $4 \%$ paraformaldehyde and
$0.2 \%$ picric acid overnight at $4^{\circ} \mathrm{C}$. Horizontal sections $(50 \mu \mathrm{m})$ were cut on a vibratome. The sections were rinsed in PBS and incubated overnight in avidin-AMCA (7-amino-4-methylcoumarin-3-acetic acid; Vector Labs, Burlingame, CA) diluted 1:200 in PBS containing 0.5\% Triton X-100. After rinsing, the sections containing the injected neurons were immunochemically labeled for VP- or OT-associated neurophysins either from semi-thin plastic sections as described previously (Armstrong et al., 1994) or by double-immunofluorescence labeling in vibratome sections. The same antibodies were used in both techniques. For double immunofluorescence, the vibratome sections were incubated in $0.5 \%$ nonfat dry milk for $1 \mathrm{hr}$ to reduce nonspecific staining, then in a cocktail of primary antibodies for $3 \mathrm{~d}$, and finally in a cocktail of secondary antibodies containing fluorescein isothiocyanate (FITC)-conjugated goat anti-rabbit and tetramethylrhodamine isothiocyanate-conjugated goat anti-mouse $\mathrm{IgG}$, for another $2 \mathrm{~d}$. VP neurons were identified with a rabbit antiserum specific for VP-neurophysin (provided by Alan Robinson, University of Pittsburgh, Pittsburgh, PA) at a 1:30,000 dilution. OT neurons were labeled with a mouse antibody PS36 (provided by Harold Gainer and Mark Whitnall, National Institutes of Health) specific for OTneurophysin at a dilution of 1:1000. All antibodies were diluted with PBS containing $0.5 \%$ Triton $\mathrm{X}-100$. Sections were mounted in a solution of glycerol-PBS (1:2), observed with a fluorescent microscope, and photographed with a Kodak Tri-X 400 film. Neurons were considered as either OT or VP types only if positive staining of one antibody was complemented by a negative reaction for the heterologous peptide.

Statistical analysis. Results were analyzed by a one-way or two-way ANOVA, followed by Tukey's $t$ test or Dunnett's $t$ test. A Student's $t$ test was used for comparing the rebound amplitude and the ratio of the firing frequency during the first second of a $4 \mathrm{sec}$ depolarizing pulse to that during the fourth. To compare the incidence of the DAP among the different experimental groups, frequencies of observations were arranged in a contingency table, and chi-square statistic was used for analysis (Zar, 1984).

\section{RESULTS}

\section{Immunohistochemistry}

Electrophysiological recordings were obtained from 86 MNCs in the SON of lactating $(n=43)$ or diestrous $(n=31)$ female rats. Of these, 39 were positive for VP-neurophysin and 35 for OTneurophysin. Neurons that were positive for both antibodies $(n=$ $2)$ or that failed to react with either antibody $(n=10)$ were not included in the analysis. For simplicity, identified neurons will be referred to hereafter as either VP or OT neurons. Figure 1 shows an example of an immunochemically labeled MNC.

\section{Passive membrane properties and $\boldsymbol{I}-\boldsymbol{V}$ relationships}

The basic membrane properties of immunoidentified MNCs from lactating and diestrous rats are shown in Table 1. Average resting membrane potential $\left(V_{\mathrm{m}}\right)$, mean input resistance $\left(R_{\mathrm{n}}\right)$, and membrane time constant $(\tau)$ did not differ among groups, either as a 
Table 1. Membrane properties of OT and VP neurons in diestrous and lactating rats

\begin{tabular}{llll} 
& $V_{\mathrm{m}}$, rest $(\mathrm{mV})$ & $R_{\mathrm{n}}(\mathrm{M} \Omega)$ & $\tau(\mathrm{msec})$ \\
\hline OT-diestrous & $-62.8 \pm 2.9(13)$ & $229.9 \pm 09.4(13)$ & $11.8 \pm 1.8(8)$ \\
OT-lactating & $-64.6 \pm 1.7(22)$ & $229.5 \pm 14.4(21)$ & $13.6 \pm 3.3(8)$ \\
VP-diestrous & $-67.7 \pm 2.4(18)$ & $257.1 \pm 20.9(18)$ & $10.8 \pm 1.6(8)$ \\
VP-lactating & $-64.5 \pm 2.1(21)$ & $223.5 \pm 16.1(21)$ & $8.6 \pm 1.4(12)$
\end{tabular}

Shown are the means \pm SEM with the numbers of neurons in parentheses. See Materials and Methods for a detailed description of the electrophysiological protocols used for measurements.

function of the neuron type or as a function of lactation. Examples of $I-V$ curves of OT and VP neurons from lactating and diestrous rats are shown in Figure 2. When most (36 of 39, or 92.3\%) VP neurons were current-clamped near approximately $-50 \mathrm{mV}$ and subjected to increasing hyperpolarizing commands, a mostly linear response was observed (Fig. 2B). In some cases, a small sag developed at the most hyperpolarized membrane potentials, probably corresponding to the activation of the hyperpolarizationactivated inward current $\left(I_{\mathrm{h}}\right)$. In contrast, most (33 of 35 , or 94.3\%) OT neurons exhibited a time- and voltage-dependent rectification, resulting in a depolarizing sag to the voltage trajectory (Fig. $2 A$ ). The rectification was evident first when the neuron was hyperpolarized a few millivolts from the holding potential, and it decreased with increasing hyperpolarization. A steady state $I-V$ curve plotted at the end of the hyperpolarizing commands revealed a strong outward rectification and a linear relationship in OT and VP neurons, respectively (Fig. 2C). The incidence of the rectification and associated RD (see below) was significantly higher in OT neurons than in VP neurons $\left(\chi^{2}=55.4 ; p=0.0001\right)$. This result is in agreement with our previous data (Stern and Armstrong, 1995a). The characteristics of this SOR observed in OT neurons recently were described in some detail (Stern and Armstrong, 1995a). Our preliminary pharmacological results (Stern and Armstrong, 1995b) indicate that the SOR is not underlain by $I_{\mathrm{h}}$ : the SOR can be isolated in the presence of 1-2 mM extracellular $\mathrm{Cs}^{+}$, which clearly blocks $I_{\mathrm{h}}$. On the other hand, the SOR but not $I_{\mathrm{h}}$ is blocked with either tetraethylammonium or $\mathrm{Ba}^{+}$. No differences between lactating or diestrous rats were found in relation to the incidence or amplitude of the rectification.

An RD with a superimposed burst of action potentials was evoked at the offset of the hyperpolarizing commands in neurons exhibiting the SOR, either in lactating or diestrous rats (see Fig. $2 A$ and Fig. 3). As reported previously (Stern and Armstrong, 1995a), with increasing hyperpolarization from approximately $-50 \mathrm{mV}$, the RD first increased in magnitude, attaining a peak at approximately $-65 \mathrm{mV}$ and decreasing thereafter. This overshoot is probably a result of the increased $R_{\mathrm{n}}$ at the end of the hyperpolarizing command (attributable to the deactivation of the SOR coupled with the slow development of the outward rectification after the hyperpolarizing command). Neurons were currentclamped at approximately $-45 \mathrm{mV}$, and the magnitude of the RD at the offset of increasing hyperpolarizing commands was measured in the presence of tetrodotoxin (TTX) (Fig. 3). Rebound peak amplitude in diestrous and lactating rats was attained at $64.6 \pm 1.3$ and $64.7 \pm 1.6 \mathrm{mV}$, respectively. The peak amplitude of the RD was significantly larger in lactating rats compared with diestrous rats $(t=2.8 ; p<0.02$; Student's $t$ test $)$.

When MNCs were current-clamped at approximately $-90 \mathrm{mV}$ and subjected to depolarizing commands, a transient outward
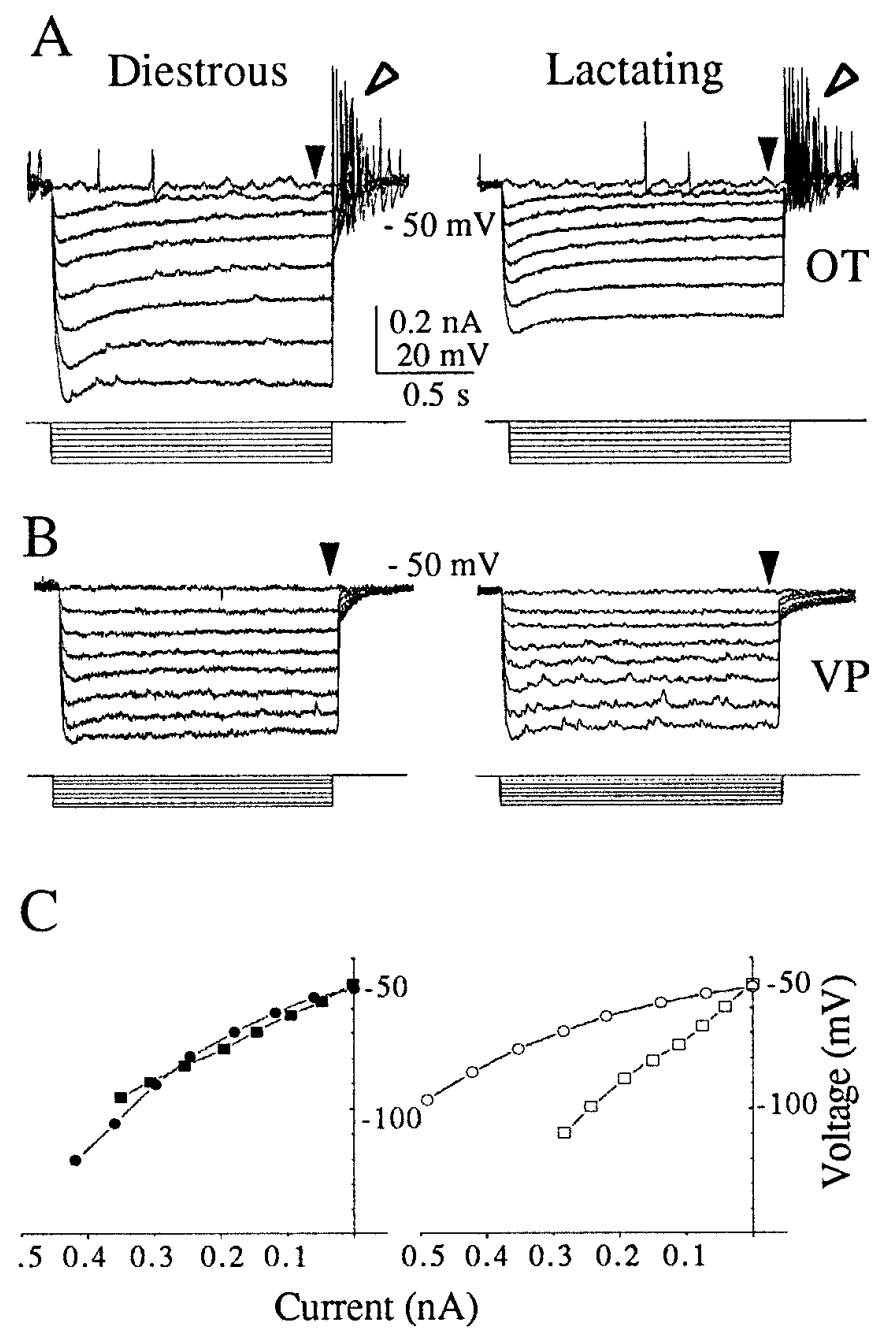

Figure 2. Examples of voltage responses to constant-current pulses for an $\mathrm{OT}$ and a VP neuron from lactating and diestrous rats. $A$, Hyperpolarizing pulses applied to OT neurons from lactating or diestrous rats revealed a time- and voltage-dependent rectification and an RD (open arrowheads). $B$, VP neurons failed to express the rectification and the RD during the hyperpolarizing pulses. $C$, Steady-state $I-V$ curves plotted at the end of the hyperpolarizing pulses (filled arrowheads in $A$ and $B$ ) revealing a strong outward rectification and a linear relationship in OT (circles) and VP neurons (squares), respectively. The difference in the slopes observed in the VP neurons shown in these examples reflects a difference in neuronal $R_{\mathrm{n}}$. Still, a linear relationship was observed in both cases. Filled and empty symbols represent diestrous and lactating rats, respectively. All traces are averages $(n=3)$.

rectification (TOR) developed, delaying the time taken for the first spike to reach threshold (Fig. $4 A$ ). An $I-V$ curve was plotted at $20 \mathrm{msec}$ after the onset of the depolarizing command, coinciding with strong development of the TOR. Examples from individual neurons are shown in Figure $4 B$. Polynomials have been shown to be a useful representation of nonlinear membrane $I-V$ relations and were used for quantitative comparisons of TOR (Jack et al., 1983; Nisenbaum and Wilson, 1995). The curves were best fitted by a third-order polynomial regression. When plotting voltage on the vertical axis and current on the horizontal axis, outward rectification is represented as a downward deflection from linearity. The negative quadratic term of the polynomial regression (present in fits from all cells) represents the degree of this downward deflection (Jack et al., 1983). A two-way ANOVA 

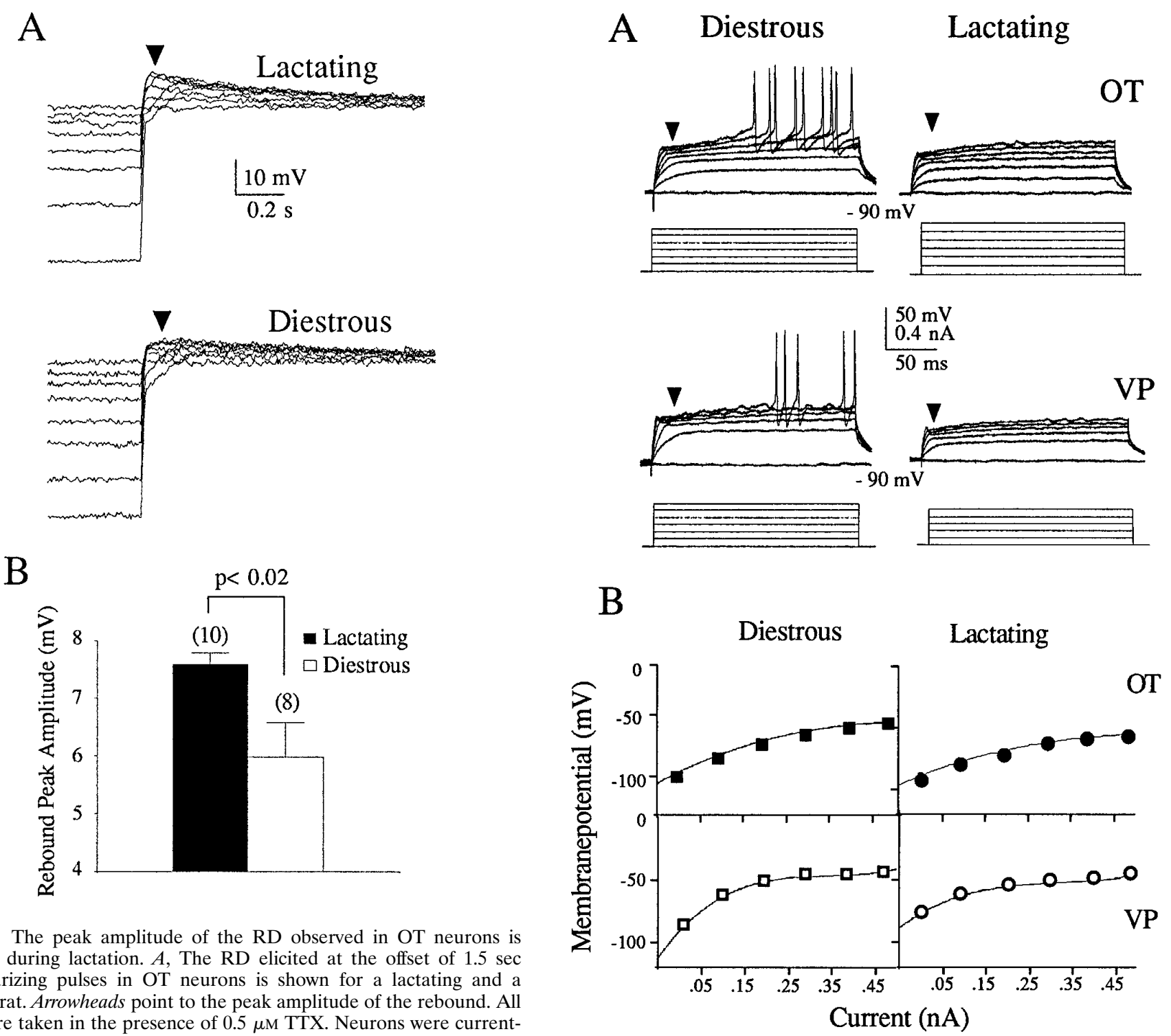

Figure 3. The peak amplitude of the RD observed in OT neurons is enhanced during lactation. $A$, The RD elicited at the offset of $1.5 \mathrm{sec}$ hyperpolarizing pulses in OT neurons is shown for a lactating and a diestrous rat. Arrowheads point to the peak amplitude of the rebound. All traces were taken in the presence of $0.5 \mu \mathrm{M}$ TTX. Neurons were currentclamped at approximately $-45 \mathrm{mV}$. $B$, The rebound peak amplitude is enhanced during lactation ( $p<0.02$; Student's $t$ test).

revealed a significantly smaller value in OT neurons $(F=4.31$; $p<0.05)$, indicating less TOR. No differences were found as a function of lactation $(F=1.8 ; p>0.05)$. Mean values $(\mathrm{M} \Omega / \mathrm{nA})$ were $1500 \pm 300(n=11)$ in OT-lactating, $2400 \pm 900(n=10)$ in VP-lactating, $1860 \pm 200(n=8)$ in OT-diestrous, and $4050 \pm$ $1000(n=10)$ in VP-diestrous rats.

\section{Action potential properties}

Spike threshold of OT neurons was on average $\sim 6 \mathrm{mV}$ more depolarized compared with VP neurons $(F=5.3 ; p<0.05)$. However, no significant differences were found when a post hoc Tukey test was applied to search for specific differences among groups.

Action potential properties were measured from spikes evoked within $5 \mathrm{mV}$ of threshold. Individual examples of spikes from the different groups are shown in Figure 5. Values for spike height, spike width, rise time, and decay time are shown in Table 2. Neurons from lactating rats displayed wider action potentials $(F=$ $9.3 ; p<0.02)$, concomitant with a slower rising time $(F=5.44$; $p<0.05)$ and a slower decay time $(F=15.6 ; p<0.001)$. When the

Figure 4. Transient outward rectification in OT and VP neurons from lactating and diestrous rats. $A$, Depolarizing pulses from a hyperpolarized holding potential induced a transient outward rectification (arrowheads), which delayed the time taken for the first spike to reach threshold. All traces except those giving rise to spikes are averages $(n=3)$. $B, I-V$ curves plotted at $20 \mathrm{msec}$ after the onset of the depolarizing pulses for neurons shown in $A$. Curves were fitted with a third-order polynomial, and the mean of the quadratic term of the polynomial regression was used to compare the degree of outward rectification (see Results). The polynomials fitted for the individual examples shown were as follows: diestrousOT: $-86.23+170 x-217 x^{2}+85.4 x^{3}, R^{2}=0.99$; diestrous-VP: $-91.1+$ $370 x-1052 x^{2}+1032 x^{3}, R^{2}=1.0$; lactating-OT: $-87.6+180 x-181 x^{2}+$ 87.1 $x^{3}, R^{2}=0.99$; lactating-VP: $-77.3+200 x-570 x^{2}+591 x^{3}, R^{2}=0.99$. The degree of the transient outward rectification, as indicated by a two-way ANOVA, was significantly smaller in OT neurons (see Results).

different groups were compared, a post hoc Tukey test indicated that these differences observed during lactation were confined to OT neurons (see Table 2). On the other hand, spike height was significantly lower in OT compared with VP neurons $(F=25.8$; $p<0.001$ ), both in lactating and in diestrous rats (see Table 2). Individual action potentials were followed by a hyperpolarizing afterpotential (HAP) (Fig. 5). The HAP mean amplitude, area, 


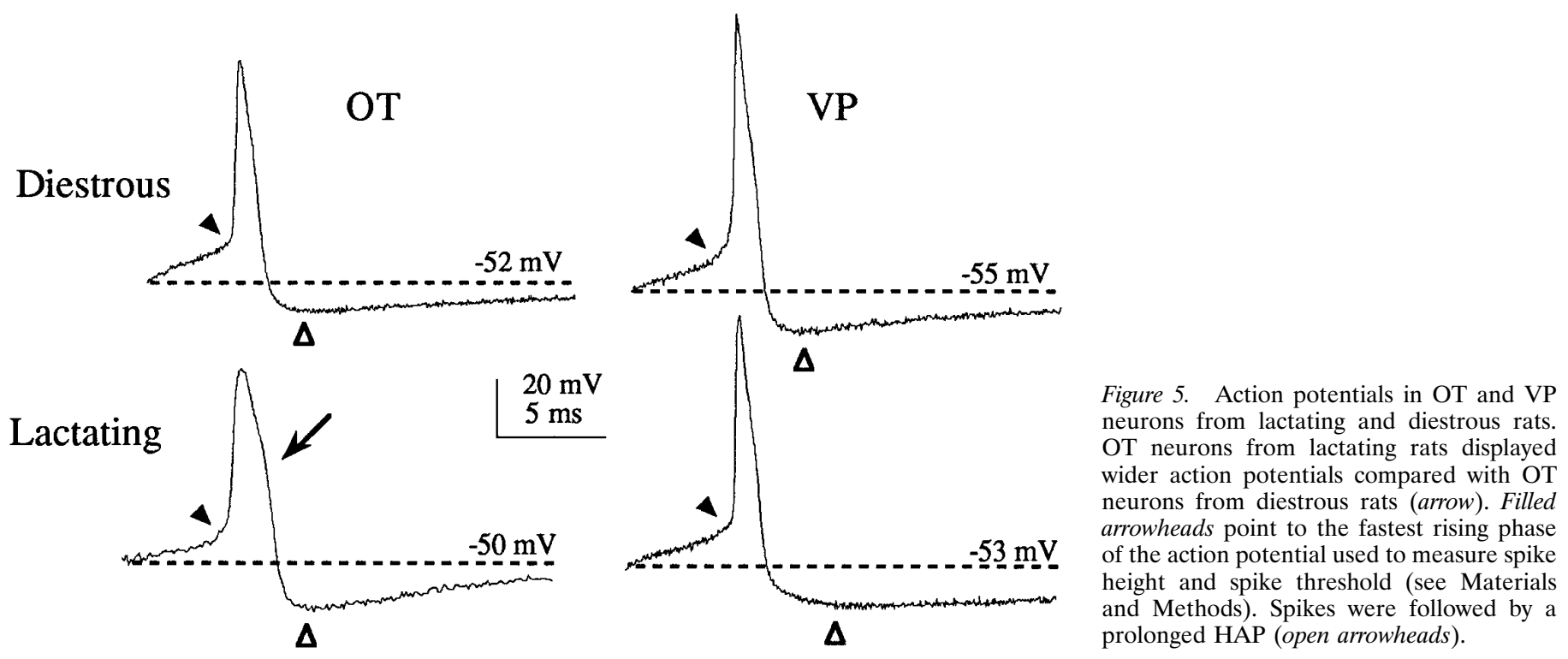

and time constant did not differ among groups (results not shown).

\section{DAP and afterhyperpolarizing potential}

The DAP is an endogenous, voltage-dependent potential thought to be involved in the generation of phasic firing in VP neurons (Andrew and Dudek, 1984a). The expression of this potential was tested by applying a train of spikes given at a frequency of $50 \mathrm{~Hz}$ while varying the membrane potential with direct current injection. Results are shown in Table 3. Neither the incidence of the DAP $\left(\chi^{2}\right.$ test $\left.=4.6 ; p=0.2\right)$ nor the mean amplitude of DAPs evoked just below spike threshold $(F=0.5 ; p=0.7$; see Table 3$)$ were significantly different among groups. Examples of DAPs are shown in Figure 6. When the neurons were depolarized enough so that action potentials were evoked on the DAP, prolonged bursts were evoked in most VP neurons. On the other hand, this response was only observed in one OT neuron.

A prominent afterhyperpolarizing potential (AHP) was observed in all MNCs after an evoked discharge of spikes (Fig. 7A). The peak amplitude and the area of the AHP were studied, and because these parameters are linearly related to the number of spikes during the train (Andrew and Dudek, 1984b; Bourque et al., 1985), the results were calculated per spike. When a $180 \mathrm{msec}$ depolarizing pulse was used to induce the AHP, the mean peak amplitude was significantly larger in neurons from lactating rats $(F=6.9 ; p<0.01)$ (Fig. $7 B)$. A post hoc Tukey test indicated that the differences were statistically significant only between OT neurons (see Fig. 7B, left panel). These differences were observed over a range of current intensities used to elicit the AHP, the results being expressed either as the AHP absolute value $(F=8.96 ; p<$ 0.01 ; ANOVA for repeated measures) or as the amplitude per spike ( $F=8.2 ; p<0.02$; ANOVA for repeated measures) (Fig. $7 C)$. Similar results were obtained when a train of two spikes (50 $\mathrm{Hz}$ ) was used to elicit the AHP (see Fig. $7 B$, right panel). The AHP area per spike also was significantly larger in neurons from lactating rats $(F=8.9 ; p<0.01)$, the differences again being restricted to OT neurons ( $p<0.05$; Tukey test). Values obtained $(\mathrm{mV} \times \mathrm{sec} / \mathrm{spike})$ were as follows: OT-diestrous, $0.19 \pm 0.04$; VP-diestrous, $0.27 \pm 0.05$; OT-lactating, $0.41 \pm 0.06$; VPlactating, $0.40 \pm 0.06$. The time course of the decay of the AHP was well fitted by a single exponential function. The time constant of this decay was significantly smaller in OT neurons compared with VP neurons, both when a constant train of pulses or a single depolarizing pulse was used ( $F=5.8$ and 5.0, respectively; $p<$ 0.05) (see Fig. 7B). However, a post hoc test failed to reveal significant differences among groups in both cases.

\section{Repetitive firing}

To further compare OT and VP neurons and to evaluate the effects of lactation, the firing response of the neurons to current pulses was studied. Frequency-current $(F-I)$ curves, spike frequency adaptation, spike broadening, and input-to-output relationship were studied in detail.

Examples of firing behavior during a $180 \mathrm{msec}$ outward current pulse $(0.4 \mathrm{nA})$ are illustrated in Figure 8. Repetitive firing was characterized by a varying degree of spike frequency adaptation observed in all tested neurons. Plots of instantaneous firing rates versus time were constructed (see insets in Fig. $8 A$ ). The decrease

Table 2. Spike properties of OT and VP neurons in diestrous and lactating rats

\begin{tabular}{llllll} 
& $\begin{array}{l}\text { Spike threshold } \\
(\mathrm{mV})\end{array}$ & Height $(\mathrm{mV})$ & Width $(\mathrm{msec})$ & Rise time $(\mathrm{msec})$ & Decay time $(\mathrm{msec})$ \\
\hline OT-diestrous & $-35.6 \pm 1.2(11)$ & $65.6 \pm 1.8^{*}(13)$ & $0.97 \pm 0.06(13)$ & $0.22 \pm 0.01(10)$ & $0.76 \pm 0.04(10)$ \\
OT-lactating & $-37.9 \pm 2.3(16)$ & $61.5 \pm 1.3^{* *}(20)$ & $1.25 \pm 0.06^{* * *(20)}$ & $0.32 \pm 0.01^{* * *(20)}$ & $1.10 \pm 0.03^{* * *}(20)$ \\
VP-diestrous & $-44.0 \pm 2.5(17)$ & $74.3 \pm 2.9(15)$ & $0.88 \pm 0.05(15)$ & $0.27 \pm 0.02(13)$ & $0.92 \pm 0.06(13)$ \\
VP-lactating & $-39.6 \pm 1.2(21)$ & $72.3 \pm 1.4(19)$ & $0.94 \pm 0.05(19)$ & $0.26 \pm 0.01(10)$ & $0.99 \pm 0.07(10)$
\end{tabular}

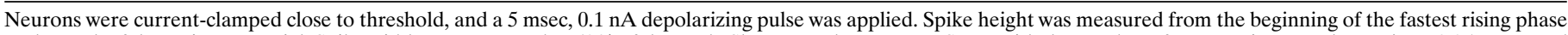

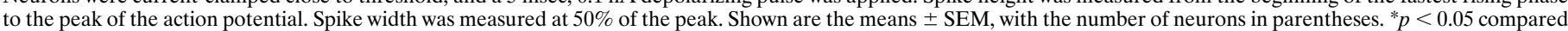
with VP-diestrous (Tukey's $t$ test); ${ }^{* *} p<0.001$ compared with VP-lactating (Tukey's $t$ test); ${ }^{* * *} p<0.01$ compared with OT-diestrous (Tukey's $t$ test). 
Table 3. DAP characteristics of OT and VP neurons in diestrous and lactating rats

\begin{tabular}{lll} 
& DAP incidence $(\%)$ & DAP amplitude $(\mathrm{mV})$ \\
\hline OT-diestrous & $50.0(5 / 10)$ & $4.28 \pm 0.7(5)$ \\
OT-lactating & $63.2(12 / 19)$ & $4.61 \pm 0.4(12)$ \\
VP-diestrous & $63.2(12 / 19)$ & $4.01 \pm 0.5(12)$ \\
VP-lactating & $87.5(14 / 16)$ & $4.74 \pm 0.5(14)$
\end{tabular}

$\overline{\text { A DAP was evoked by applying a train of two spikes at a frequency of } 50 \mathrm{~Hz} \text {. Shown }}$ are the means \pm SEM with the number of neurons in parentheses. A $\chi^{2}$ test indicated no significant differences in the incidence of the DAP among groups (4.6, $p>0.05)$.

of the firing rates was best fitted by a double exponential curve, and the slowest adaptation time constants were calculated and used for comparisons. As shown in Figure 8B, OT neurons exhibited a significantly slower adaptation time constant compared with VP neurons, both in lactating and diestrous rats $(F=36 ; p<$ $0.0001)$. No significant differences were observed between lactating and diestrous rats $(F=0.34 ; p>0.05)$. On the other hand, the first interspike interval frequency of these evoked trains was significantly decreased as a function of lactation $(F=68.7 ; p<$ 0.0001 ) both in OT and VP neurons (see Figs. $8 C, 9 A$ ). This resulted in a shift of the adaptation curve to a lower frequency level in lactating animals.

$F-I$ plots were constructed for the first three interspike intervals (Fig. $9 A$, only the first interspike interval is shown). In all cases, faster firing rates were observed as the current pulses were made larger. The increase in firing frequency was well fitted by a single exponential curve, and the steepness of the response was calculated and compared among groups. As shown in Table 4, neurons from diestrous rats showed a steeper $F-I$ response compared with neurons from lactating rats $(F=12.9,17$, and 18.6 for first, second, and third interspike intervals, respectively; $p<0.01$ ). These differences were significant both in OT and VP neurons (see Table 4).

The input-output relation during repetitive firing was studied by plotting the number of spikes versus the current amplitude during the application of outward current pulses of increasing amplitude (Fig. 9B). The evoked firing response was significantly suppressed during lactation in OT $(F=4.8 ; p<0.05)$ but not in VP neurons ( $F=3.7 ; p>0.05$; ANOVA for repeated measures) (Fig. 9B). Another characteristic of MNCs during repetitive firing is the progressive increment in spike duration (spike broadening) (Andrew and Dudek, 1985; Bourque and Renaud, 1985b). Figure $9 C$ shows a plot of the mean spike duration for the first nine spikes during a $180 \mathrm{msec}$ depolarizing pulse $(0.4 \mathrm{nA})$ for OT and VP neurons from lactating and diestrous rats. All supraoptic neurons showed spike broadening. When comparisons were made between diestrous and lactating rats, an ANOVA for repeated measures indicated that all spikes in the train were broader during lactation in OT $(F=74 ; p<0.0001)$ but not in VP neurons $(F=2.4 ; p>$ $0.05)$. Furthermore, significant interactions were observed between OT neurons $(F=2.5 ; p<0.02)$, indicating that the curves were not parallel.

Because of the slowly developing and sustained characteristics of the SOR (Stern and Armstrong, 1995a), prolonged firing discharges are expected to be affected. To explore the possible correlation between the expression of this property and firing behavior, a long depolarizing pulse (4 sec duration) was applied, and the firing response of SOR-positive and SOR-negative neurons was evaluated (Fig. 10). During this time frame, the SOR is fully developed, and the adaptation attributable to the AHP (which has a time constant $<600 \mathrm{msec}$ ) has peaked. Neurons lacking the expression of SOR displayed a sustained firing response during the $4 \mathrm{sec}$ depolarizing pulse, with no significant change of the discharge frequency as a function of time. On the other hand, most neurons expressing SOR initially responded with a high-frequency, long burst of action potentials, followed by short clusters or isolated action potentials (see Fig. 10A). In one case, the neuron stopped firing after $\sim 2 \mathrm{sec}$ of depolarization. The mean firing frequency per second during the prolonged depolarization was plotted (Fig. 10B) and analyzed by a two-way ANOVA

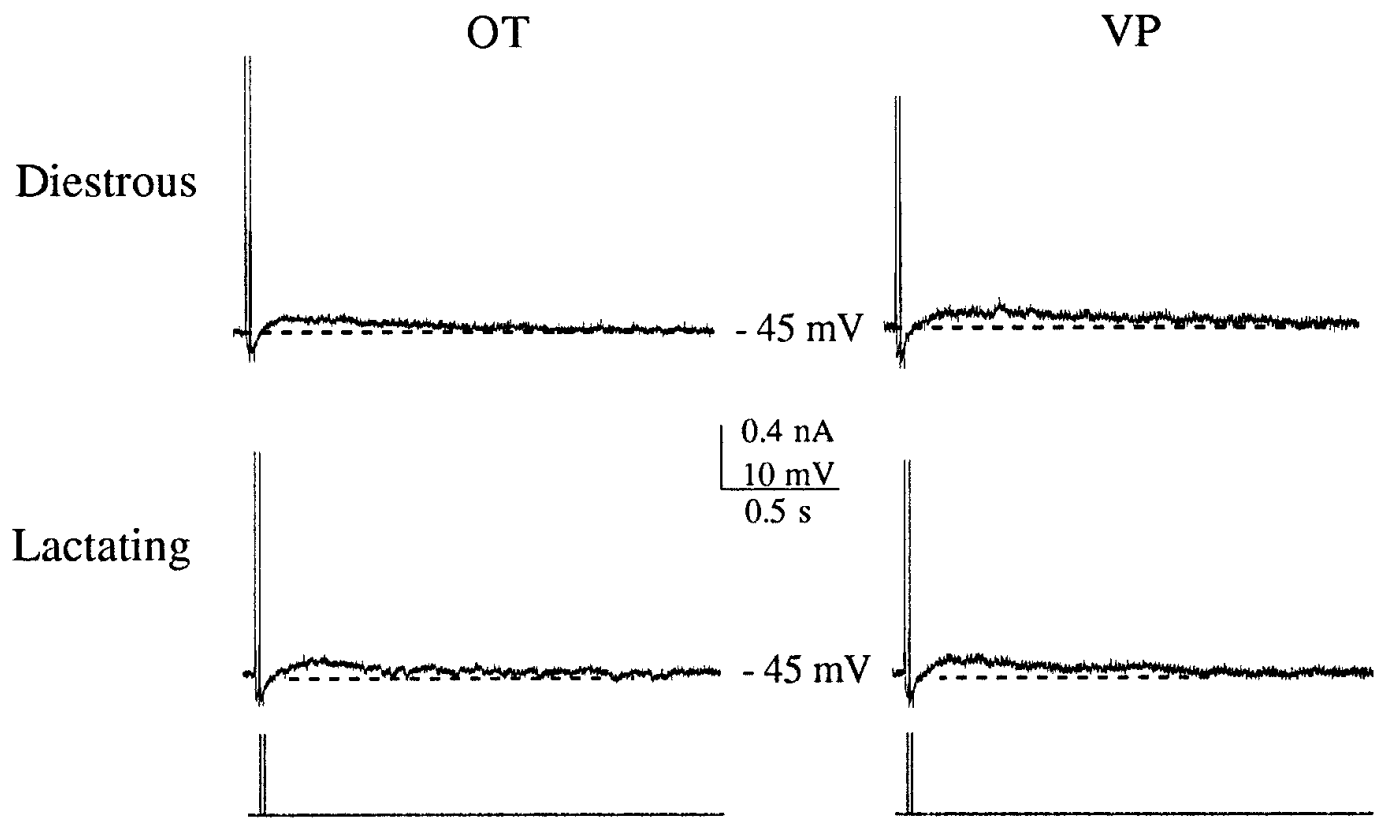

Figure 6. DAP in OT and VP neurons from lactating and diestrous rats. DAPs were elicited both in OT and VP neurons after a train of two spikes at $50 \mathrm{~Hz}$. No significant differences were observed among groups regarding DAP amplitude or incidence (see Table 3). Spike amplitude has been clipped. 

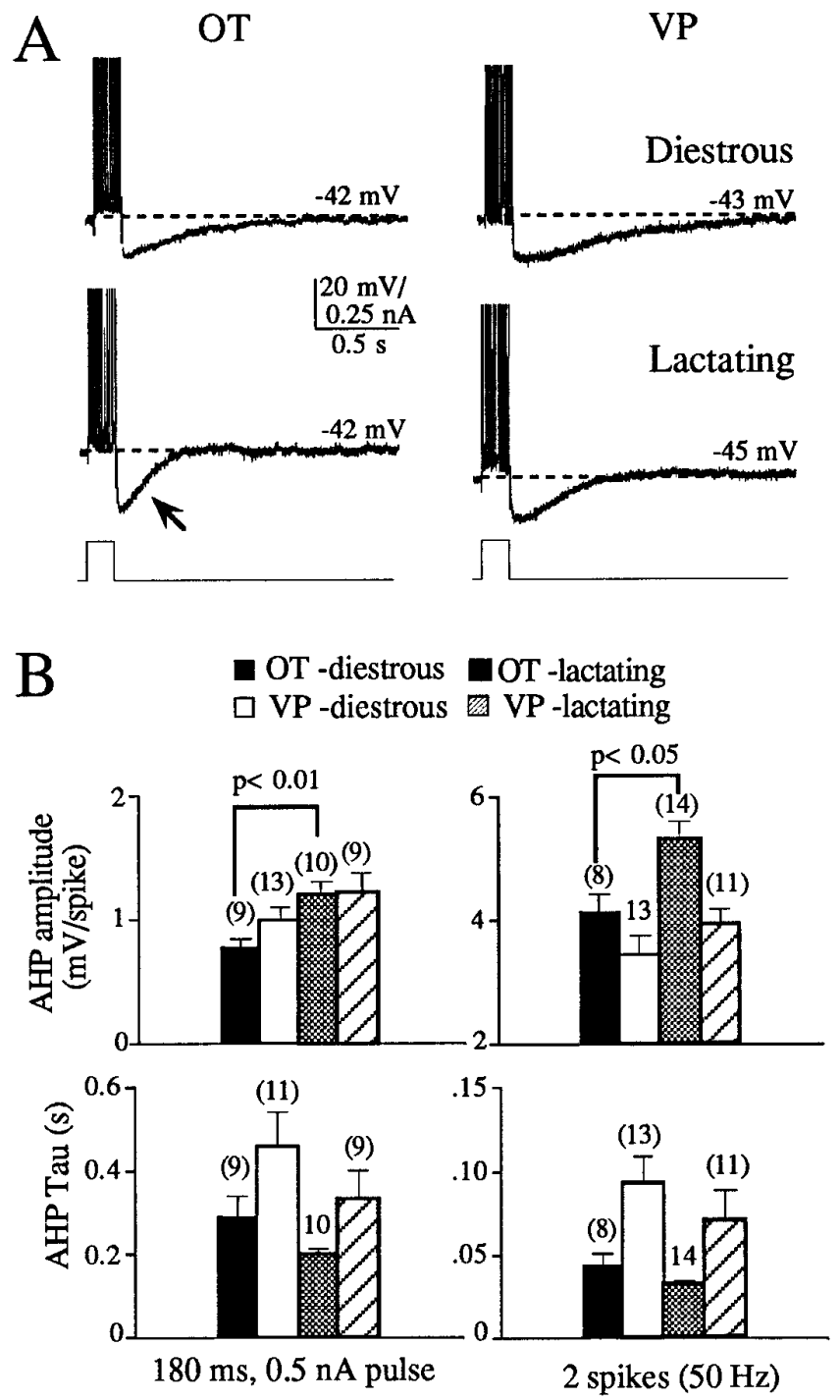

$\mathrm{C}$

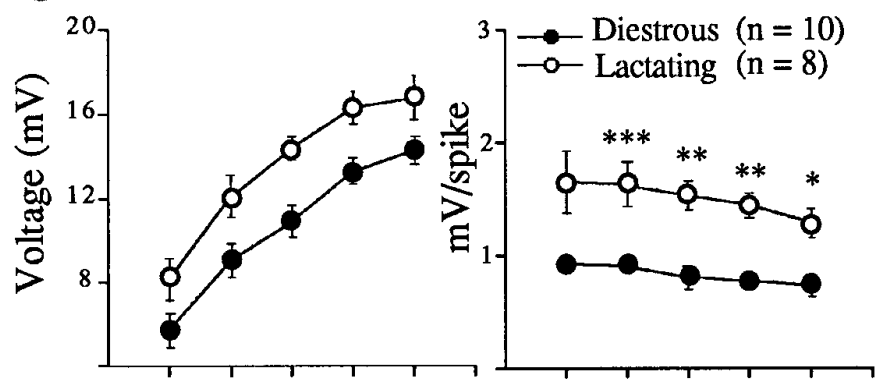

Figure 7. AHP in OT and VP neurons from lactating and diestrous rats. $A$, Individual examples of AHPs from OT and VP neurons from diestrous and lactating rats are shown (arrow). The AHP was evoked by a rectangular, $180 \mathrm{msec}$ depolarizing pulse. $B$, The amplitude and time course of the AHP evoked either by a rectangular pulse (left panel) or by a train of two spikes (right panel) at $50 \mathrm{~Hz}$ are compared among groups. OT neurons from lactating rats exhibited a larger AHP in both conditions compared with OT neurons from diestrous rats. Also, the time constant of the AHP's decay was significantly faster in OT compared with VP neurons in both stimulation protocols used. $C$, The AHP amplitude of OT neurons from lactating rats was significantly larger than that of OT neurons from diestrous rats over a range of currents used to elicit the AHP. Left panel shows the AHP amplitude expressed in absolute value $(\mathrm{mV})$; in the right panel, results are expressed per spike (mV/spike). ${ }^{*} p<0.02,{ }^{*} p<0.01$, $* * * p<0.001$ compared with lactating rats (Tukey's $t$ test). for repeated measures. SOR-positive neurons showed a significantly slower firing rate compared with SOR-negative neurons $(F=10.6 ; p<0.01)$. The firing frequency decreased significantly as a function of time $(F=17.6 ; p<0.0001)$, but significant differences were observed only in SOR-positive neurons (Dunnett's test, see Fig. 10B). Thus, the ratio of the firing frequency during the first second of the depolarizing pulse to that during the fourth second was significantly higher in neurons expressing the SOR $(t=2.5 ; p<0.05$; Student's $t$ test) (see Fig. 10C).

\section{DISCUSSION}

The main objectives of the present study were to characterize the electrical properties of SON VP and OT neurons in female rats and to determine whether these properties are altered during lactation. To our knowledge, this is the first detailed study of the electrophysiological properties of immunoidentified SON neurons during lactation.

During late pregnancy, parturition, and lactation, SON neurons have been shown to undergo a variety of morphological changes, including an increase in the amount of somatosomatic apposition, dendritic bundling, double synapses, and dye-coupling (Hatton et al., 1987; Hatton, 1990). Furthermore, a rearrangement of SON synaptic inputs involving an increase of GABAergic innervation has been described (Gies and Theodosis, 1994). Although these results provide strong evidence for a structural plasticity in the SON during lactation, the presence of changes in the intrinsic electrical properties of these neurons has never been studied in detail.

As reported previously in female (Stern and Armstrong, 1995a) and male rats (Armstrong et al., 1994), the mean values for $V_{\mathrm{m}}$, $R_{\mathrm{n}}$, and $\tau$ did not differ between OT and VP neurons, either in lactating or diestrous rats. However, in females, OT, but not VP, neurons expressed an SOR and an RD (Stern and Armstrong, 1995a). The incidence and magnitude of the SOR was not changed during lactation. However, the RD peak amplitude was significantly larger in lactating rats. Our preliminary investigations using protocols similar to those used in this study indicate that OT neurons in male rats also exhibit SOR and RD (our unpublished observations).

The expression of the RD might be influenced and even prevented by the activation of a transient outward current $\left(I_{\mathrm{A}}\right)$ at similar membrane potentials (Pape et al., 1994). The presence of a TOR in SON neurons has been studied previously under voltage clamp and represents a transient, $\mathrm{K}^{+}$current such as $I_{\mathrm{A}}$ (Bourque, 1988; Cobbett et al., 1989; Nagamoto et al., 1995). However, neurons were not immunoidentified, and the possible modulatory effects of lactation were not assessed. As shown in the present study, TOR was expressed by SON neurons, probably because of activation of $I_{\mathrm{A}}$. Because the TOR was significantly smaller in OT compared with VP neurons, the expression of the RD in OT neurons might be facilitated by the activation of a smaller TOR at the offset of the hyperpolarizing commands. These results suggest that a difference in the size and/or in the voltage-dependence of $I_{\mathrm{A}}$ exists between OT and VP neurons. Interestingly, it was previously shown that only $\sim 50 \%$ of acutely SON-dissociated neurons expressed the transient outward current (O'Regan and Cobbett, 1993). Voltage-clamp studies of $I_{\mathrm{A}}$ from identified MNCs should resolve whether this current differs between OT and VP subtypes.

The activation of an SOR at subthreshold levels might significantly affect membrane spike threshold and excitability by providing a repolarizing drive during slow depolarization, as shown for the expression of M-current in sympathetic ganglia (Adams et al., 


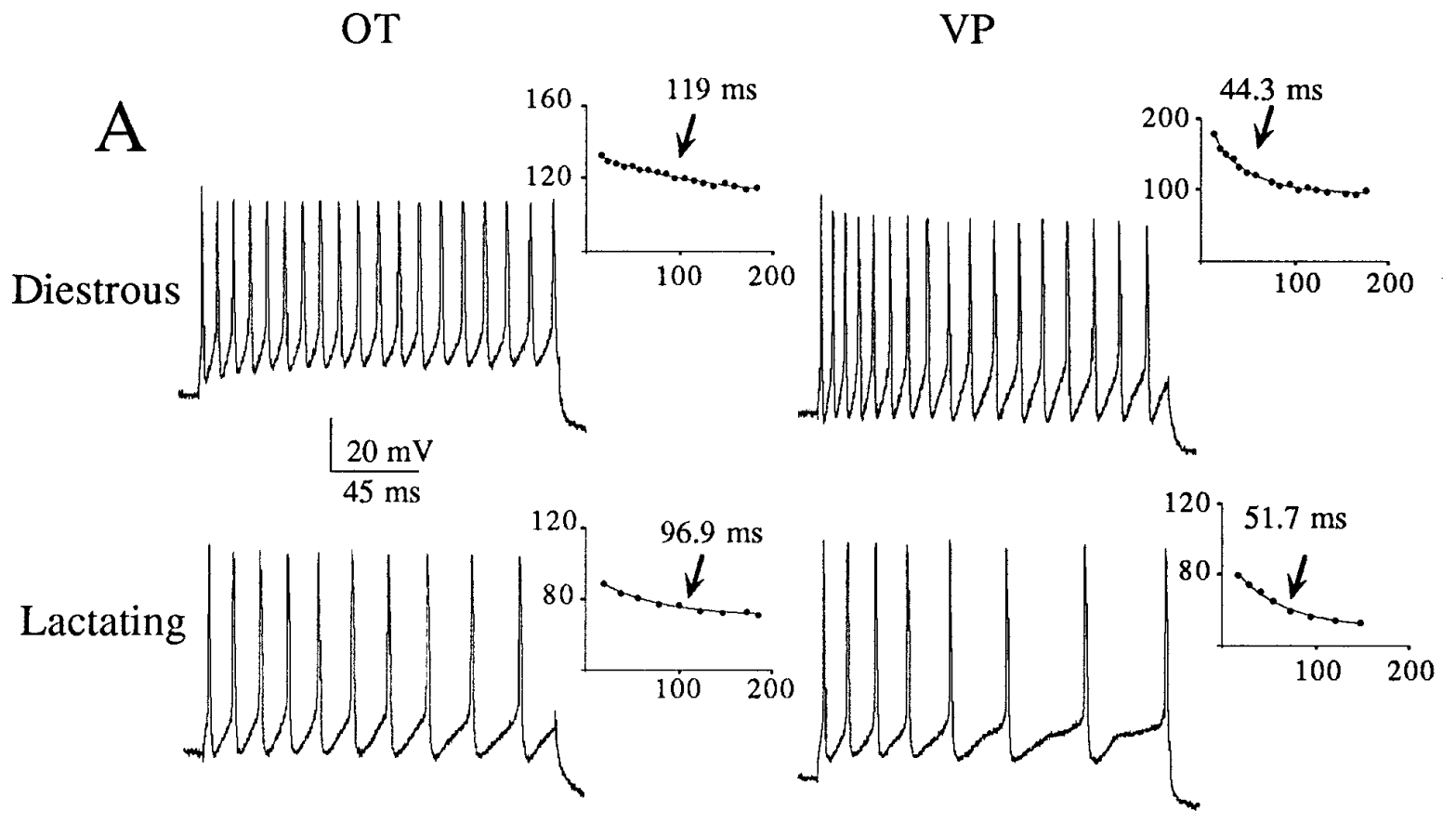

B

Time course of spike frequency adaptation

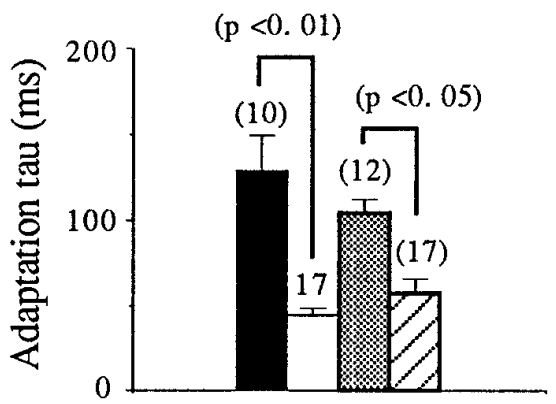

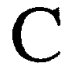

First interspike interval frequency

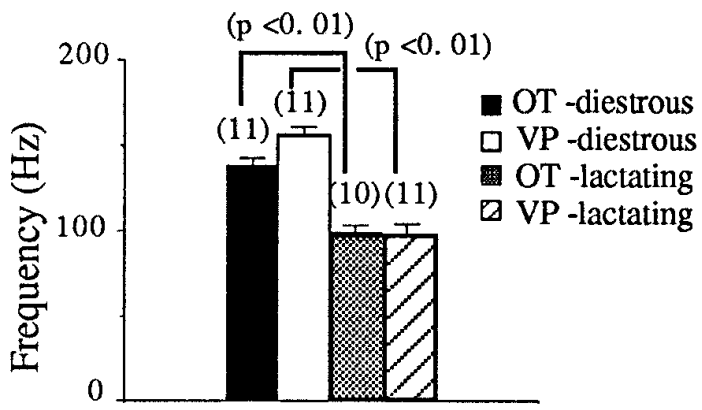

Figure 8. Spike frequency adaptation during repetitive firing in OT and VP neurons from lactating and diestrous rats. $A$, A varying degree of spike frequency adaptation was observed in all tested neurons during a $180 \mathrm{msec}$ outward current pulse $(0.4 \mathrm{nA})$. Insets show plots of instantaneous firing rates $(\mathrm{Hz})$ versus time $(\mathrm{msec})$. The slowest time constant obtained after fitting a double exponential function is also shown. $B$, The adaptation time constant was significantly slower in OT compared with VP neurons, both in lactating and diestrous rats. $C$, The first interspike interval frequency was significantly decreased in lactating rats compared with diestrous rats, both in OT and VP neurons. The number of neurons is in parentheses. Spike amplitudes have been clipped.

1982). In fact, we observed that the spike threshold of OT neurons was significantly more depolarized than that of VP neurons. Furthermore, neurons expressing the SOR showed a decreased firing response as a function of time during prolonged depolarization. Thus, it might be argued that when OT neurons are depolarized, the activation of the SOR decreases neuronal excitability, raising spike threshold and limiting repetitive firing. These results don't rule out that cell type differences in other properties as well may also account for the firing differences observed. The expression of the RD coupled to the SOR might facilitate the expression of short burst responses in OT neurons. Short bursts would be further facilitated during lactation, because of the enhanced RD observed during this stage. Alternatively, if OT neurons are sufficiently depolarized, inhibitory inputs that transiently hyperpolarize the neuron and deactivate the SOR may induce a burst of action potentials. It was recently shown that GABA facilitated the bursting activity of OT neurons during lactation while inhibiting background activity (Moos, 1995). Furthermore, $\mathrm{GABA}_{\mathrm{A}}$ antagonists interrupted bursting (Moos, 1995; Voisin et al., 1995). These results suggest that GABAergic inputs play an important role in the maintenance of the mechanisms underlying the milk-ejection reflex. We suggest that one of the mechanisms whereby GABA may facilitate bursting is by deactivating the SOR, which after termination of the inhibitory input would be followed by an overshoot of action potentials, as stated above.

The phasic firing pattern in VP neurons is thought to be generated by the summation of DAPs (Andrew and Dudek, 1983, 1984a). Furthermore, it has been argued that the DAP is exclusively displayed by VP neurons (Bourque, 1989). We found that $57 \%$ of OT and $76 \%$ of VP neurons exhibited DAPs. In male rats, 


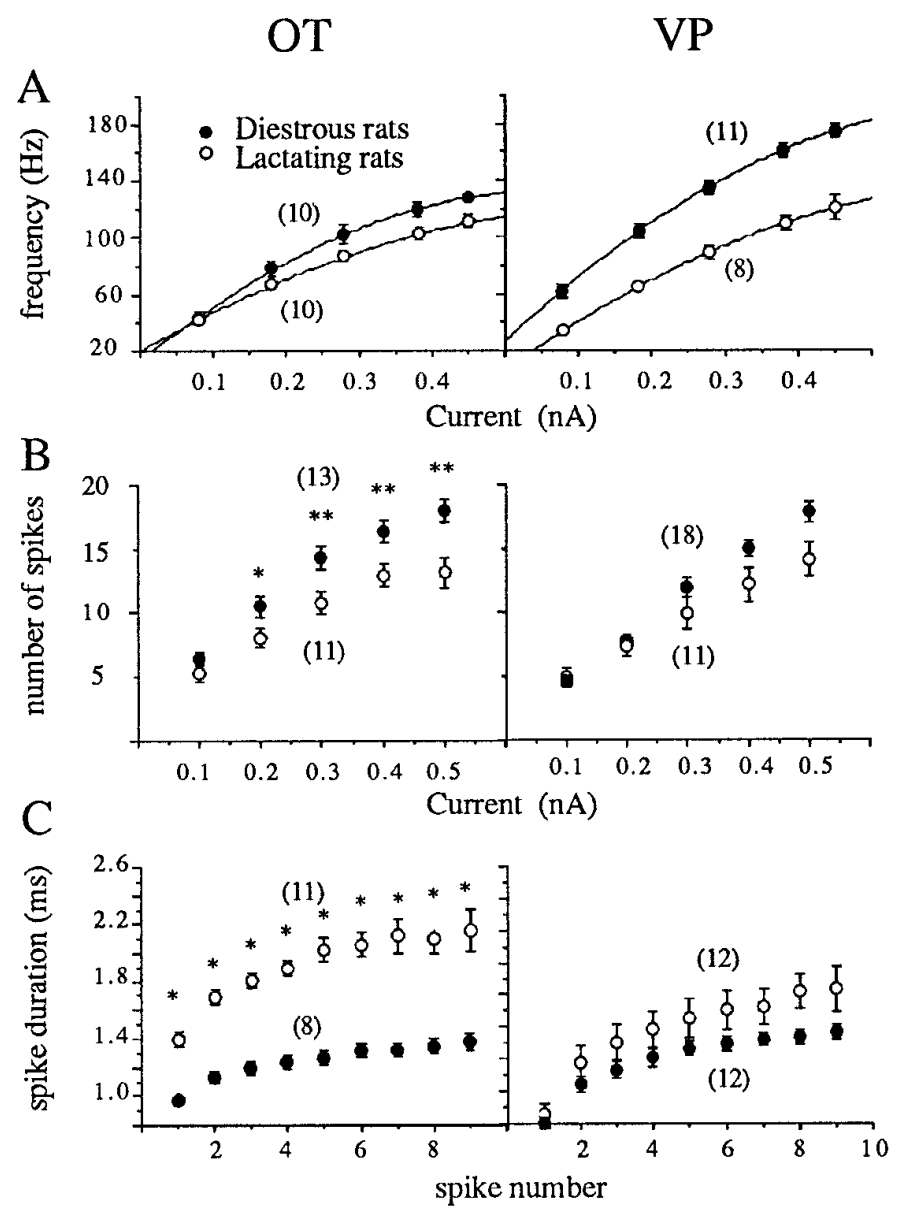

Figure 9. $F-I$ plots, input-output relation, and spike broadening in OT and VP neurons from lactating and diestrous rats. $A, F-I$ plots for the first interspike interval during a $180 \mathrm{msec}$ outward current pulse. The firing rates incremented as the current pulses were made larger. The steepness of the response was calculated after fitting the curves with a single exponential function (see Table 4). B, Plot of the mean number of spikes versus current amplitude during the application of outward current pulses of increasing amplitude. OT neurons from lactating rats displayed a decreased response compared with OT neurons from diestrous rats. ${ }^{*} p<$ $0.02,{ }^{* *} p<0.001$ (Tukey's $t$ test). $C$, Plot of the mean spike duration versus the number of spikes in the train during a $180 \mathrm{msec}$ outward current pulse $(0.4 \mathrm{nA})$. Spike broadening was observed in all tested neurons. However, the increment in spike duration was significantly larger in OT neurons from lactating rats compared with OT neurons from diestrous rats (ANOVA for repeated measures, $F=74 ; p<0.0001$; interactions, $F=2.5 ; p<0.02) ;{ }^{*} p<0.001$ (Tukey's $t$ test). The number of neurons is in parentheses for all figures.

about one-third of OT neurons displayed DAPs, but only rarely exhibited phasic firing (Armstrong et al., 1994). Although the incidence of phasic firing wasn't examined in the present study, the results did suggest that prolonged discharges (i.e., beyond the time course of the DAP) were rarely observed after inducing a DAP in OT neurons. These results suggest that the presence of a DAP per se cannot account for the differences in phasic activity between VP and OT neurons. Nevertheless, the expression of the DAP in OT neurons would facilitate short bursts of activity.

Action potentials of OT neurons were significantly changed during lactation. Wider spikes and slower rising and decay times were observed. The precise mechanisms underlying these changes were not addressed in the present study. However, regardless of the mechanism involved (i.e., a larger $\mathrm{Ca}^{2+}$ inward current and/or
Table 4. $F-I$ relationship in OT and VP neurons in diestrous and lactating rats

\begin{tabular}{llll} 
& $\begin{array}{l}\text { ISI } 1 F-I \\
\text { steepness } \\
(\mathrm{Hz} / \mathrm{nA})\end{array}$ & $\begin{array}{l}\text { ISI } 2 F-I \\
\text { steepness } \\
(\mathrm{Hz} / \mathrm{nA})\end{array}$ & $\begin{array}{l}\text { ISI } 3 F-I \\
\text { steepness } \\
(\mathrm{Hz} / \mathrm{nA})\end{array}$ \\
\hline OT-diestrous & $421.7 \pm 47^{*}(9)$ & $437.0 \pm 42^{*}(9)$ & $436.4 \pm 60^{*}(9)$ \\
OT-lactating & $294.8 \pm 23(7)$ & $274.8 \pm 18(7)$ & $276.8 \pm 17(7)$ \\
VP-diestrous & $513.0 \pm 18^{* *}(13)$ & $504.8 \pm 23^{*}(15)$ & $601.1 \pm 33(15)$ \\
VP-lactating & $381.1 \pm 53(7)$ & $352.9 \pm 68(7)$ & $355.2 \pm 55(7)$
\end{tabular}

$\overline{F-I \text { plots were constructed for the first three interspike intervals during a } 180 \mathrm{msec}}$ outward current pulse of varying intensity. The steepness of the response was calculated by fitting a single exponential curve. Shown are the means \pm SEM with the number of neurons in parentheses. * $p<0.05$ compared with OT-lactating (Tukey's $t$ test); ${ }^{* *} p<0.01$ compared with VP-lactating (Tukey's $t$ test). ISI, Interspike interval.

a change in $\mathrm{Na}^{+}$and/or $\mathrm{K}^{+}$currents), an increase in $\mathrm{Ca}^{2+}$ influx per spike, through high-threshold channels, is expected in this situation. Spike threshold values of SON neurons have been infrequently reported in the literature. However, when noted, a more hyperpolarized level compared with our present results was reported in male rats (Bourque and Renaud, 1985a).

That an increase in $\mathrm{Ca}^{2+}$ influx per spike occurs in OT neurons during lactation is supported by our finding that the magnitude of the AHP, a $\mathrm{Ca}^{2+}$-dependent potential (Andrew and Dudek, 1984a; Bourque et al., 1985), was larger in OT neurons from lactating rats. Our results suggest that individual spikes are contributing more to the AHP during lactation.

The present results indicate that during short $(180 \mathrm{msec}) \mathrm{de}-$ polarizations, OT neurons adapt less than VP neurons. Frequency adaptation over this time course is related to the AHP (Bourque and Brown, 1987). Although the AHP amplitude was not significantly different between neuron types, the time constant of the AHP's decay was faster in OT neurons. On the other hand, OT neurons from lactating rats showed a decreased initial firing frequency during a depolarizing pulse, coinciding with a larger AHP amplitude already visible after a train of two spikes (see Fig. $7 B$ ). These results would argue that the time course of the AHP and its peak amplitude influence the time course of spike frequency adaptation and the initial spike frequency during trains of action potentials, respectively. Because the AHP is known to limit firing by providing intrinsic inhibition, strong depolarizations during lactation would be followed by a stronger stabilizing effect on spike firing.

Significant changes in repetitive firing were observed in neurons from lactating rats, which were selective to or more profound in OT neurons. A shallower $F-I$ slope for early interspike intervals and a decreased input-output relation during depolarizing pulses imply that OT neurons during lactation have a diminished gain in the transduction of depolarizing events into action potentials firing. The larger AHP found in OT neurons during lactation might contribute to these findings. The decreased input-output relation, besides favoring itself the expression of short bursts, might eventually attenuate the tonic activation of OT neurons that might counteract their bursting activity. Interestingly, it has been shown that the release of OT to hypovolemia (Köehler et al., 1993), hyperosmolality, and stress (Higuchi et al., 1988) is diminished during lactation. These stimuli have been shown to tonically activate OT neurons (Brimble and Dyball, 1977; Poulain et al., 1977; Wakerley et al., 1978).

SON neurons show spike broadening during repetitive firing 
Figure 10. Firing response during prolonged depolarization in neurons with or without the SOR. $A$, Examples of firing response during a long depolarizing pulse $(4 \mathrm{sec})$ in an SORpositive (left trace) and an SOR-negative (right trace) neuron. Note the decrease in firing rate observed in the SOR-positive compared with the SOR-negative neuron during the depolarizing pulse. $B$, Plot of mean firing frequency per second for SOR-positive and SOR-negative neurons. The firing frequency decreased significantly as a function of time in SOR-positive neurons $\left({ }^{*} p<0.001\right.$, compared with the firing frequency during the first second of the depolarizing pulse; Dunnett's $t$ test). $C$, Ratio of the firing frequency during the first second of the depolarizing pulse to that during the fourth second in SOR-positive and SOR-negative neurons. ${ }^{* *} p<0.05$ (Student's $t$ test), $n=8$ and 6 for SOR-negative and SOR-positive neurons, respectively.
A SOR positive
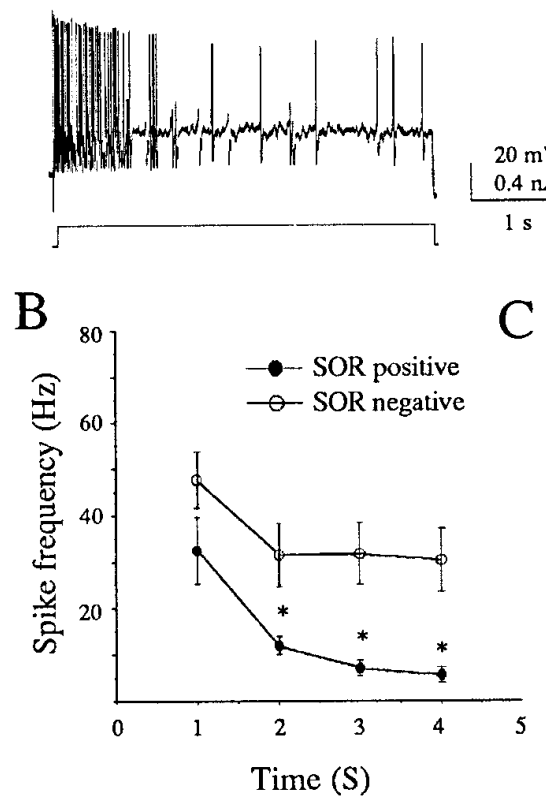

$20 \mathrm{mV}$

$1 \mathrm{~s}$
SOR negative

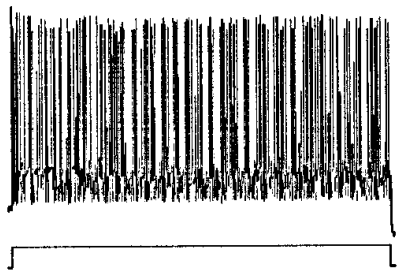

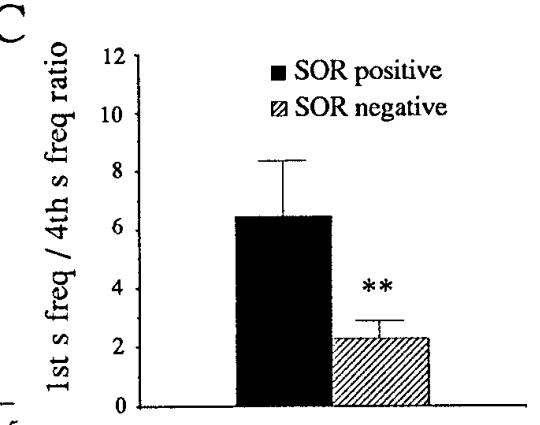

(Andrew and Dudek, 1985; Bourque and Renaud, 1985b). Frequency-dependent changes in $\mathrm{K}^{+}$and $\mathrm{Ca}^{2+}$ conductances have been implicated in the expression of spike broadening (O'Regan and Cobbett, 1993). Our results indicate that spike broadening was significantly enhanced in OT neurons from lactating rats. These results, taken together with the presence of wider spikes and larger $\mathrm{Ca}^{2+}$-dependent AHPs in OT neurons during lactation, suggest that spike-induced $\mathrm{Ca}^{2+}$ influx is selectively increased in these neurons during lactation. This enhanced $\mathrm{Ca}^{2+}$ influx could directly bear on the somatodendritic release of OT shown to occur during lactation (Moos et al., 1989) and which facilitates the OT neuron burst discharge (Lambert et al., 1993).

\section{REFERENCES}

Adams P, Brown DA, Constanti A (1982) M-currents and other potassium currents in bullfrog sympathetic neurons. J Physiol (Lond) 330:537-572.

Aghajanian GK, Rasmussen K (1989) Intracellular studies in the facial nucleus illustrating a simple new method for obtaining viable motoneurons in adult rat brain slices. Synapse 3:331-338.

Andrew RD, Dudek FE (1983) Burst discharge in mammalian neuroendocrine cells involves an intrinsic regenerative mechanism. Science 221:1050-1052.

Andrew RD, Dudek FE (1984a) Analysis of intracellularly recorded phasic bursting by mammalian neuroendocrine cells. J Neurophysiol 51:552-566.

Andrew RD, Dudek FE (1984b) Intrinsic inhibition in magnocellular neuroendocrine cells of rat hypothalamus. J Physiol (Lond) 353: 171-185.

Andrew RD, Dudek FE (1985) Spike broadening in magnocellular neuroendocrine cells of rat hypothalamic slices. Brain Res 334:176-179.

Armstrong WE, Stern JE (1995) Changes in the electrophysiological properties of oxytocin and vasopressin neurons during lactation. Soc Neurosci Abstr 21:1148.

Armstrong WE, Smith BN, Tian M (1994) Electrophysiological characteristics of immunochemically identified oxytocin and vasopressin neurons in vitro. J Physiol (Lond) 475:115-128.

Bicknell R (1988) Downstream consequences of bursting activity in oxytocin neurones. In: Pulsatility in neuroendocrine systems (Leng G, ed), pp 62-74. Boca Raton: CRC.
Bicknell R, Flint APF, Leng G, Sheldrick EL (1982) Phasic pattern of electrical stimulation enhances oxytocin secretion from the isolated neurohypophysis. Neurosci Lett 30:47-50.

Bourque CW (1988) Transient calcium-dependent potassium current in magnocellular neurosecretory cells of the rat supraoptic nucleus. J Physiol (Lond) 397:331-347.

Bourque CW (1989) Ionic basis for the intrinsic activation of rat supraoptic neurones by hyperosmotic stimuli. J Physiol (Lond) 417:263-277.

Bourque CW, Brown DA (1987) Apamin and $d$-tubocurarine block the afterhyperpolarization of rat supraoptic neurosecretory neurons. Neurosci Lett 82:185-190.

Bourque CW, Renaud LP (1985a) Calcium-dependent action potentials in rat supraoptic neurosecretory neurones recorded in vitro. J Physiol (Lond) 363:419-428.

Bourque CW, Renaud LP (1985b) Activity dependence of action potential duration in rat supraoptic neurosecretory neurones recorded in vitro. J Physiol (Lond) 363:429-439.

Bourque CW, Randle CR, Renaud LP (1985) Calcium-dependent potassium conductance in rat supraoptic nucleus neurosecretory neurons. J Neurophysiol 54:1375-1382.

Brimble M, Dyball REJ (1977) Characterization of the responses of oxytocin- and vasopressin-secreting neurones in the supraoptic nucleus to osmotic stimulation. J Physiol (Lond) 271:253-271.

Cazalis M, Dayanithi G, Nordmann JJ (1985) The role of patterned burst and interburst interval on the excitation-coupling mechanism in the isolated rat neural lobe. J Physiol (Lond) 369:45-60.

Cobbett P, Legendre P, Mason WT (1989) Characterization of three types of potassium current in cultured neurones of rat supraoptic nucleus area. J Physiol (Lond) 410:443-462.

Dreifuss J-J, Kalnins I, Kelly JS, Ruf KB (1971) Action potentials and release of neurohypophysial hormones in vitro. J Physiol (Lond) 220:87-103.

Dutton A, Dyball REJ (1979) Phasic firing enhances vasopressin release from the rat neurohypophysis. J Physiol (Lond) 290:443-440.

Gies U, Theodosis T (1994) Synaptic plasticity in the rat supraoptic nucleus during lactation involves GABA innervation and oxytocin neurons: a quantitative immunocytochemical analysis. J Neurosci 14:2861-2869.

Hatton GI (1990) Emerging concepts of structure-function dynamics in adult brain: the hypothalamo-neurohypophysial system. Prog Neurobiol 34:437-504.

Hatton GI, Yang QZ, Cobbett P (1987) Dye coupling among immunocytochemically identified neurons in the supraoptic nucleus: increased incidence in lactating rats. Neuroscience 21:923-930. 
Higuchi T, Honda K, Takano S, Negoro H (1988) Reduced oxytocin response to osmotic stimulus and immobilization stress in lactating rats. J Endocrinol 116:225-230.

Jack JJB, Noble D, Tsien RW (1983) Electrical current flow in excitable cells. Oxford, UK: Oxford UP.

Kita H, Armstrong WE (1991) A biotin-containing compound $\mathrm{N}$-(2aminoethyl) biotinamide for intracellular labeling and neuronal tracing studies: comparison with biocytin. J Neurosci Methods 37: 141-150.

Köehler EM, McLemore GL, Tang W, Summy-Long JY (1993) Osmoregulation of the magnocellular system during pregnancy and lactation. Am J Physiol 264:555-560.

Lambert RC, Moos F, Richard PH (1993) Action of endogenous oxytocin within the paraventricular or supraoptic nuclei: a powerful link in the regulation of the bursting pattern of oxytocin neurons during the milk-ejection reflex in rats. Neuroscience 57:1027-1038.

Moos FC (1995) GABA-induced facilitation of the periodic bursting activity of oxytocin neurones in suckled rats. J Physiol (Lond) 488:103-114.

Moos FC, Poulain DA, Rodriguez F, Guerne Y, Vincent JD, Richard P (1989) Release of oxytocin within the supraoptic nucleus during the milk ejection reflex in rats. Exp Brain Res 76:593-602.

Nagamoto T, Inenaga K, Yamashita H (1995) Transient outward current in adult rat supraoptic neurones with slice patch-clamp technique: inhibition by angiotensin II. J Physiol (Lond) 485:87-96.

Nisenbaum ES, Wilson CJ (1995) Potassium currents responsible for inward and outward rectification in rat neostriatal spiny projection neurons. J Neurosci 15:4449-4463.

O'Regan MH, Cobbett P (1993) Somatic currents contribute to frequency-dependent spike-broadening in supraoptic neuroendocrine cells. Neurosci Lett 161:169-173.
Pape H, Budde T, Mager R, Kisvarday ZF (1994) Prevention of $\mathrm{Ca}^{2+}$ mediated action potentials in GABAergic local circuit neurons of rat thalamus by a transient K current. J Physiol (Lond) 478:403-422.

Poulain D, Wakerley JB, Dyball REJ (1977) Electrophysiological differentiation of oxytocin- and vasopressin-secreting neurones. Proc R Soc Lond [Biol] 196:367-384.

Rice M, Nicholson C (1991) Diffusion characteristics and extracellular volume fraction during normoxia and hypoxia in slices of rat neostriatum. J Neurophysiol 65:264-272.

Smith B, Armstrong WE (1990) Tuberal supraoptic neurons. I. Morphological and electrophysiological characteristics observed with intracellular recording and biocytin filling in vitro. Neuroscience 38:469-483.

Stern JE, Armstrong WE (1995a) Electrophysiological differences between oxytocin and vasopressin neurons recorded from female rats in vitro. J Physiol (Lond) 488:701-708.

Stern JE, Armstrong WE (1995b) Electrophysiological differences between oxytocin and vasopressin neurons in female rats. Soc Neurosci Abstr 21:1148.

Voisin DL, Herbison AE, Poulain DA (1995) Central inhibitory effects of muscimol and bicuculline on the milk ejection reflex in the anaesthetized rat. J Physiol (Lond) 483:211-224.

Wakerley JB, Lincoln DW (1973) The milk-ejection reflex of the rat: a 20- to 40-fold acceleration in the firing of paraventricular neurones during oxytocin release. J Endocrinol 57:477-493.

Wakerley J, Poulain DA, Brown D (1978) Comparison of firing patterns in oxytocin- and vasopressin-releasing neurons during progressive dehydration. Brain Res 148:425-440.

Zar JH (1984) Biostatistical analysis. Englewood Cliffs, NJ: PrenticeHall. 